\title{
Recurrent 17 bp duplication in PITX3 is primarily associated with posterior polar cataract (CPP4)
}

\author{
V Berry*, Z Yang*, P K F Addison, P J Francis, A lonides, G Karan, L Jiang, W Lin, J Hu, R Yang, \\ A Moore, K Zhang, S S Bhattacharya
}

J Med Genet 2004;41:e109 (http://www.jmedgenet.com/cgi/content/full/41/8/e109). doi: 10.1136/jmg.2004.020289

$\mathrm{T}$ he human lens is a unique tissue continually evolving and ever increasing in size primarily formulated by placement of newly differentiated fibre cells in concentric lamellae. This is analogous to an onion skin arrangement. This differentiation triggers loss of both nucleus and other cell organelles. Furthermore, the main factors for maintaining lens clarity are the tight and ordered mutual binding of the fibre cells and the packing of their intracellular building blocks termed crystallins. It is known that lens transparency is a crucial trade-off between the concentration of these macromolecules and their hydration. Disruption of normal levels of hydration can lead to opacity.

Cataract, opacification of the eye lens, is the commonest cause of blindness in the world. During infancy and early childhood it frequently results in visual impairment or blindness. In children, it can cause an irreversible amblyopia. Nearly half of all congenital cataracts are normally characterised as familial and they are perceived to be a key feature if not the predominant feature of about 200 genetic diseases. ${ }^{1-3}$ Recent research has paved the way for a better understanding of the underlying mechanisms of inherited forms of cataract. Inherited cataract is clinically heterogeneous, and, thus far, more than 14 distinct loci in humans have been identified, for 11 phenotypically distinct forms of autosomal dominant congenital cataracts (ADCC). ${ }^{4}$

Progress has been made in elucidating cataract causing mutations in human genes encoding the transparent intracellular lens proteins (crystallins), membrane gap junction proteins (connexins), water channel proteins (aquaporins), developmental transcription factors FOXE $3{ }^{6}$ EYAl, ${ }^{7}$ PAX6, ${ }^{8}$ MAF $^{9}$ and PITX $3 .{ }^{10}$ Based on this insight, it can be deduced that cataracts exhibit marked phenotypic and genotypic heterogeneity.

Here we report a number of interesting findings about the PITX3 gene: (1) we have found mutations in this gene in three different ethnic populations: English, Chinese and Hispanic; (2) a novel mutation has been found in the Hispanic family; and (3) we have found a unique recurrent 17 bp duplication in the $3^{\prime}$ end of the gene. This is a hotspot for posterior polar cataract and ASMD in four unrelated families. Finally, we have defined a novel locus 10q25 encompassing PITX3 for posterior polar cataract (CPP4).

\section{METHODS \\ Phenotyping}

Using the registers at Moorfields Eye Hospital, London, we traced three large families with posterior polar cataract, two of which included a small number of individuals with anterior segment mesenchymal dysgenesis (ASMD). Two further families with posterior polar cataract were obtained through the clinical service affiliated to the University of Utah, UT, USA. All family members underwent comprehensive clinical and ophthalmic examination. Particular

\section{Key points}

- Molecular genetic studies of five unrelated families with autosomal dominant posterior polar cataract are described. Three families are of English descent, one is of Hispanic descent, and one is of Chinese origin. A recurrent 17 bp duplication in PITX3 occurred in four of the families, two of which also had anterior segment mesenchymal dysgenesis (ASMD). We also report a fifth family with posterior polar cataract only with a novel deletion mutation in the same gene, PITX3.

- Sequence analysis of this gene revealed, in exon 4 , a 17 bp duplication (657-673dup17) that cosegregated with all the affected members of four families. It resulted in a frameshift in codon 220 and produced an aberrant protein consisting of 94 additional residues. Further sequence analysis of PITX3 in the Hispanic family revealed a novel deletion mutation, 650delG, in exon 4, which cosegregated with disease. The mutations found in the PITX3 gene affect regions outside the homeodomain in the C-terminal end of the protein and result primarily in posterior polar cataract and, in two families, also ASMD.

- Our data indicate the existence of a new locus for posterior polar cataract termed CPP4.

attention was given to cataract morphology and anterior segment examination by direct slit lamp examination and photography. Autosomal dominant inheritance of the cataract was supported by the presence of affected individuals in each of the generations, equal numbers of affected males and females, and male-to-male transmission.

\section{Genotyping and linkage analysis}

Genomic DNA was extracted from EDTA sequestered blood samples taken with informed consent and local ethical approval using the Nucleon II DNA extraction kit (Tepnel Life Sciences).

Individuals were genotyped using fluorescently labelled primers to amplify microsatellite markers and analysed using an ABI Prism 3100 Genetic Analyzer (Applied Biosystems). Two-point linkage analysis was carried out using the MLINK program from the LINKAGE v. 5.10 software package. ${ }^{11}$ We carried out pedigree and haplotype construction using Cyrillic v. 2.1.3 software.

Abbreviations: ADCC, autosomal dominant congenital cataracts; $A S M D$, anterior segment mesenchymal dysgenesis 


\section{Sequencing of the PITX3 gene}

Genomic DNA from all the individuals was amplified using PCR Reddy Mix (AB gene) and PITX3-specific primers. Samples were processed through 30 cycles of amplification consisting of $1 \mathrm{~min}$ at $94^{\circ} \mathrm{C}$ (denaturation), $1 \mathrm{~min}$ at $60^{\circ} \mathrm{C}$ (annealing), and $1 \mathrm{~min}$ at $72^{\circ} \mathrm{C}$ (extension). The final step was lengthened to $5 \mathrm{~min}$. Direct sequencing of PCR products was performed using the BigDye Terminator Cycle Sequencing Ready Reaction Kit (Applied Biosystems) on a fluorescent sequencer (ABI 3100) in accordance with the manufacturer's instructions.

\section{RESULTS}

A four generation family (fig 1A) of English descent with autosomal dominant posterior polar cataract underwent a full clinical examination. The opacity (fig 2A), which was bilateral in all cases, consisted of a single well-defined plaque that was confined to the posterior pole of the lens. Hospital records indicated that usually the opacity was present either at birth or developed during the first few months of life and progressed, with age, to other regions of the lens. One individual of this family III: 5 had severe ASMD in addition to posterior polar cataract (fig 2A and B). All other affected members of this family had only posterior polar cataract and no other ocular or systemic abnormalities.

Linkage analysis was performed in this family (A) and, after the exclusion of a number of candidate loci, we obtained a positive two-point LOD score (3.91 at recombination fraction 0) for the marker DloS192, flanked by D10S1686 and D10S1693, encompassing the PITX3 locus.

Another four-generation family (fig 1B) of English descent with posterior polar cataract was investigated. Out of 11 affected individuals, four also had ASMD (II:2, III:3, IV:1, and IV:2). No further clinical abnormalities were detected. Furthermore, three large families of English (fig 1C), Chinese (fig 1D), and Hispanic (fig IE) descent were thoroughly

A

II

III

IV

।

II

III

IV

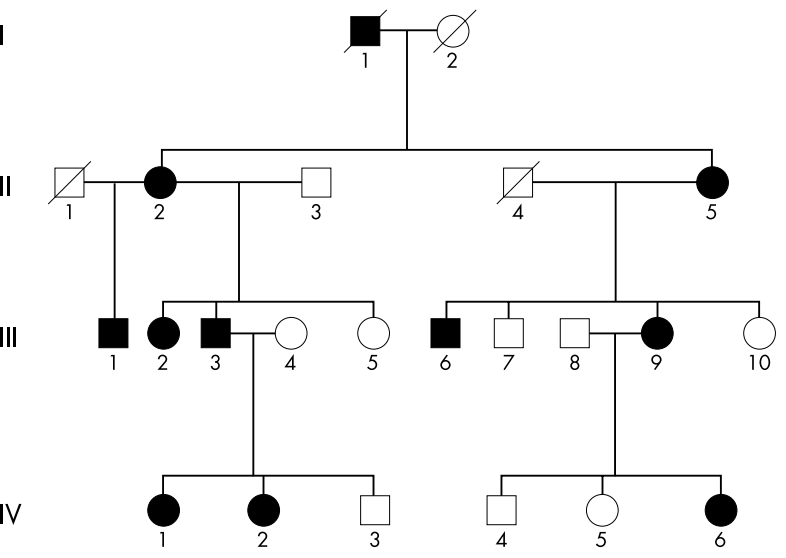


C

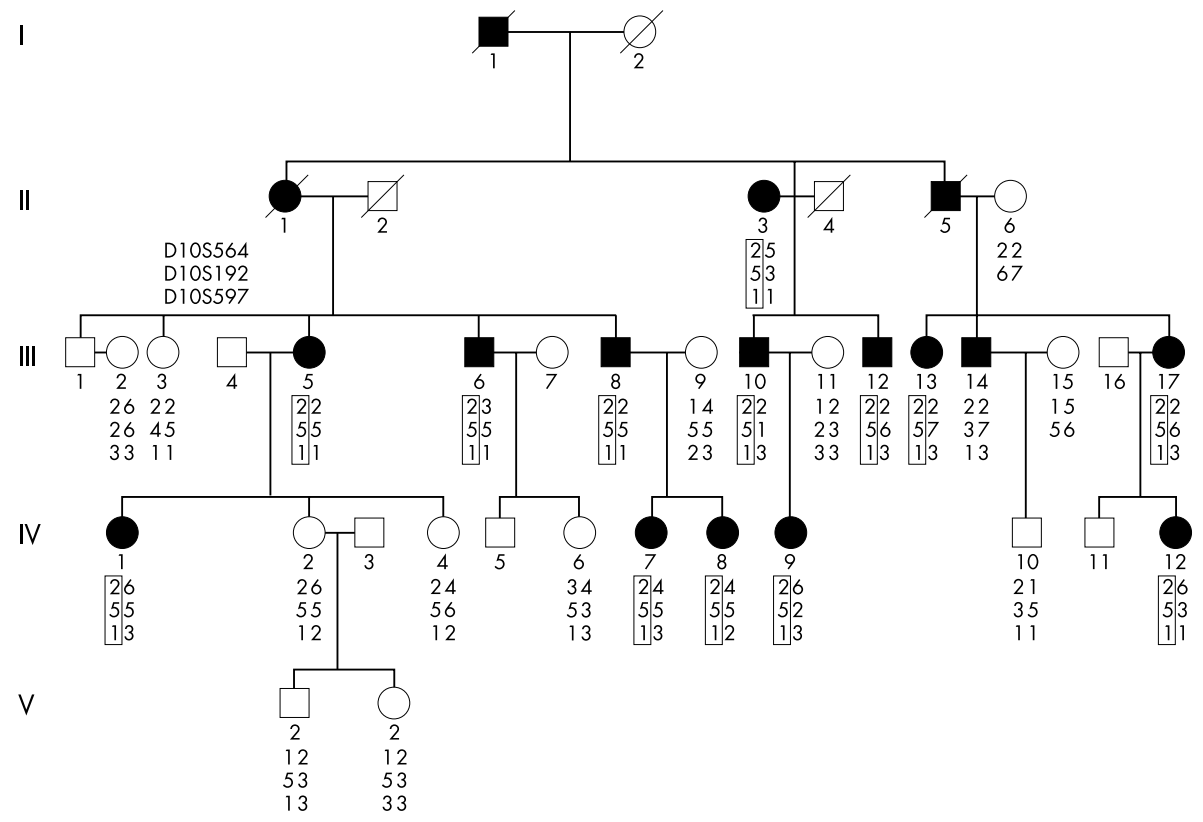

D

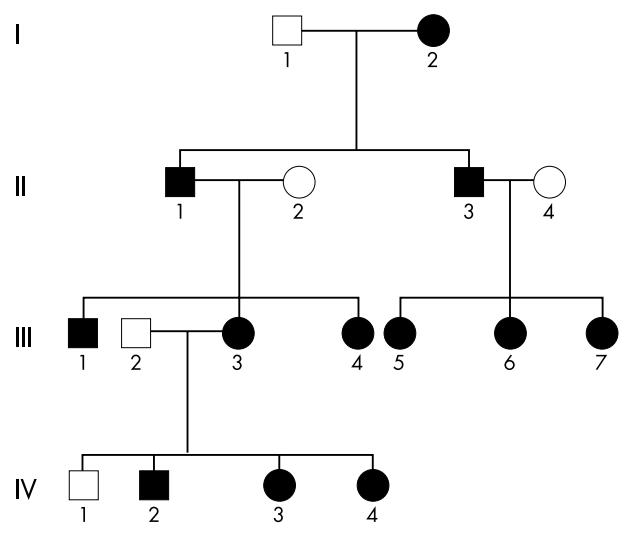

$\mathrm{E}$

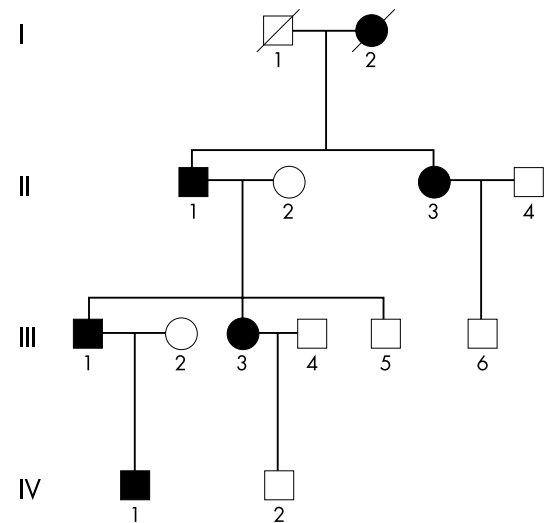

Figure 1 Pedigrees: (A), (B), (C), (D), and (E) with CPP4. Square symbols denote males; circles denote females; affected individuals are denoted by black symbols. Autosomal dominant inheritance is suggested both by the presence of affected males and females in each generation and by male-tomale transmission. Families (A) and (C) are shown with haplotype data.

examined. All affected individuals had only progressive posterior polar cataract and had no other abnormalities. All
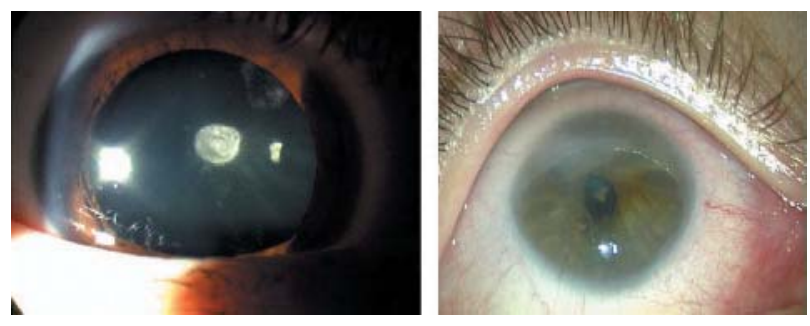

Figure 2 (A) Direct illumination slit lamp photograph of posterior polar cataract observed in a 15 year old female (IV:7) in the family with CPP4. (B) Direct illumination photograph showing ASMD in a 41 year old female (III:5) in family A. five families were linked to chromosome 10q25 with mutations identified in the PITX3 gene.

PITX 3 comprises four exons and encodes a protein of 302 amino acid residues. Sequence analysis of this gene revealed, in exon 4, a 17 bp duplication (657-673dup17; fig 3A and C) that cosegregated with all the affected members of four families (A-D). It resulted in a frameshift in codon 220 and produced an aberrant protein consisting of 94 additional residues. Further sequence analysis of PITX3 in family $\mathrm{E}$ revealed a novel deletion mutation $650 \mathrm{delG}$ (fig $3 \mathrm{~B}$ and $\mathrm{C}$ ) in exon 4, which cosegregated with disease. The mutations found in the PITX3 gene affect regions outside the homeodomain in the C-terminal end of the protein and result primarily in CPP4 and, in two families, also ASMD. This region is thought to be involved in complex protein-protein interactions, imparting specificity and efficiency to homeoprotein function. ${ }^{12}$ These mutations were not seen in 100 healthy individuals. 


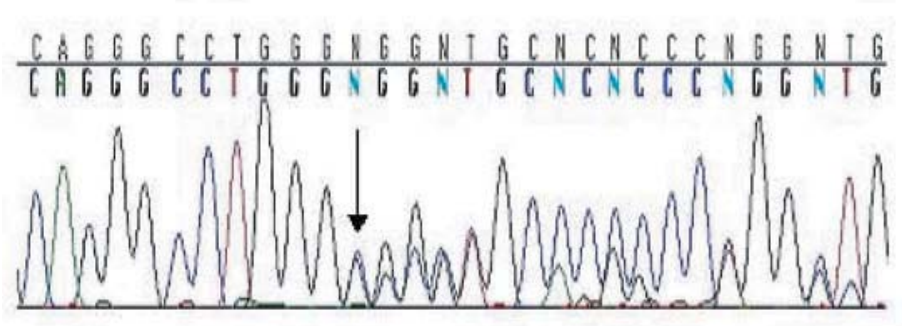

:TGGGGCCCTG CAGGCCCT GGICCGGGG OCCCCCCCG GICCT GUCCT

PITX3 Normal
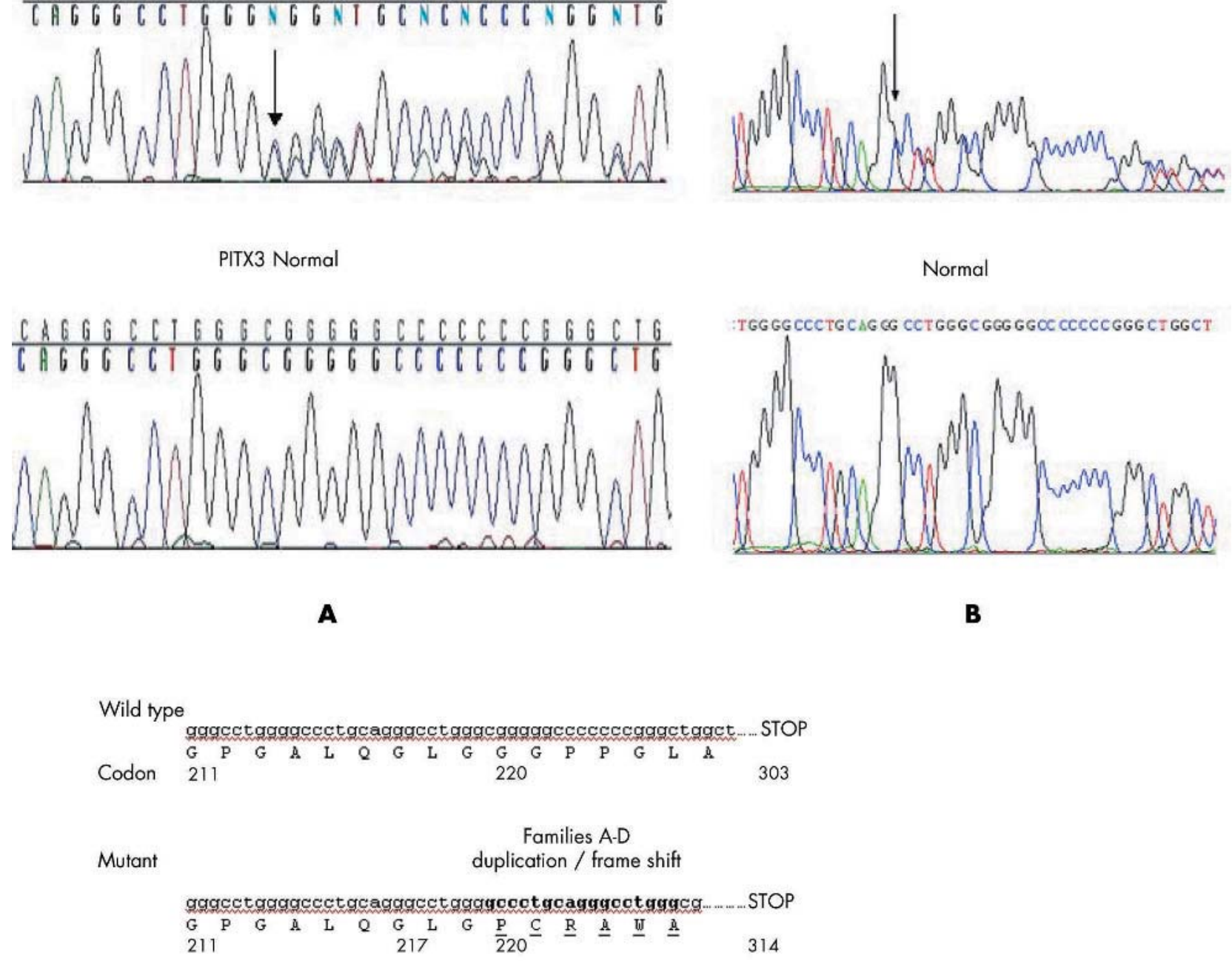

Wild type

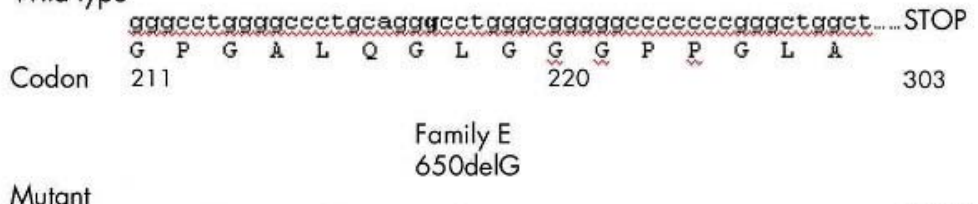

Mutant

650delG

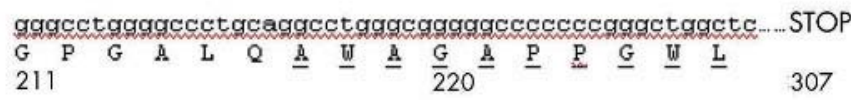

C

Figure 3 (A) Sequence analysis of PITX3 gene showing 17 bp duplication in an affected individual and (B) an affected individual of family E showing a deletion mutation in the PITX3 gene. (C) A fragment of PITX 3 sequence in a normal and in an affected individual where the nucleotide duplication and deletion are shown in boldface.

\section{DISCUSSION}

Posterior polar cataract is a clinically distinct opacity that is located at the back of the lens and, because of its proximity to the optical centre of the eye, can have a marked effect on visual acuity. Initially, dominantly inherited posterior polar cataract was linked to the haptoglobin locus on chromosome 16q. ${ }^{13}$ Subsequently, further loci for posterior polar cataract were mapped: CPP1 on $1 \mathrm{p} 36,{ }^{14} \mathrm{CPP} 2$ on $11 \mathrm{q} 21,{ }^{15}$ and CPP3 on chromosome 20pl2. ${ }^{16}$ We now define CPP4 on the long arm of chromosome 10, encompassing the PITX3 gene, a 
member of the transcriptional regulator family (PITX3, MIM 602669).

In the last few years a number of mutations in transcription factors have been shown to cause ASMD and cataracts: PAX6, FOXE3, EYA1, MAF, and PITX3. PAX6 mutations were identified in heterogeneous anterior-segment malformations in a family including Rieger and Peter anomalies. FOXE3 was found to be expressed in the anterior lens epithelium and to be mutated in patients with ocular disorders. Three novel missense mutations in the EYAl gene have been identified in patients who had congenital cataracts and ASMD. Mutations in $M A F$, a basic region leucine zipper (bZIP) transcription factor, which is expressed in vertebrate lens development and regulates the expression of the eye lens crystallins, cause cataract and also ASMD.

Unlike the aforementioned transcription factors, PITX 3 can cause isolated cataracts without ASMD. We report this phenomenon in three large unrelated families. PITX3 encodes a paired-like class of homeobox transcription factor, a member of the PITX family of genes which also includes PITX1 and PITX2. PITX2 and PITX3 genes are involved in eye development as they are expressed in most developing tissues of the eye, such as cornea, lens, and retina. ${ }^{17}$ In the aphakia mouse mutant, two deletions in the promoter of the homeobox transcription factor Pitx3 lead to loss of its function and to arrest of eye development at the lens stalk stage. ${ }^{18}$ Mutations in the homologous human PITX3 gene have been demonstrated to cause cataracts and anterior segment dysgenesis. ${ }^{10}$

Here we show that posterior polar cataract (CPP4) is indeed the predominant feature of PITX3 mutations. The 17 bp duplication mutation in the PITX3 gene is a hotspot for posterior polar cataract since it has been seen in our four unrelated families (established by haplotype analysis, data not presented) of different ethnic groups. Two of the families also had a few individuals with ASMD. Additionally, we have found a novel deletion mutation (650delG) in the same region of PITX3 which results in CPP4 alone.

Earlier the same $17 \mathrm{bp}$ duplication mutation was reported in a large family with ASMD and cataract. ${ }^{19}$ It was reported as an insertion but sequence analysis indicates it is a duplication in the $3^{\prime}$ end of the coding sequence, resulting in a frameshift. That family was previously reported to be linked to the MNS blood group on the long arm of chromosome $4 .^{20}$ These findings showed that the affected individuals from the ASMD family had cortical cataracts of differing severity. The authors found a second mutation in the same gene in a small family with isolated congenital total cataract. ${ }^{10}$ This suggested that cataract, and not ASMD, may be the major feature of PITX3 mutations.

Our data show that the PITX 3 gene plays a larger role in lens development than in the development of the rest of the anterior segment. Hence, mutations in this gene lead in all affected individuals to cataract. Only five out of 50 affected individuals (from only two of the five families) had ASMD in addition to cataract. In fact, out of these two families, in one family only one individual had ASMD and her affected child with the same 17 bp duplication mutation had only cataract. Based on our findings, it may be possible that another gene could cause ASMD.

The mutations found in the PITX3 gene affect regions outside the homeodomain in the C-terminal end of the protein and result in different phenotypes-either cataract alone or cataract combined with ASMD. This region is thought to be involved in complex protein-protein interactions, imparting specificity and efficiency to homeoprotein function. ${ }^{12}$ Functional studies are required to provide further insights into the mechanisms giving rise to the specific manifestations of these mutations.

\section{ACKNOWLEDGEMENTS}

We thank Dr Naushin H Waseem for her suggestions. We would like to thank the members of the families for taking part in this study.

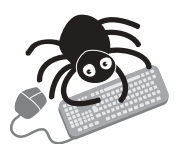

The accession number and URL for data in this article are as follows: Online Mendelian Inheritance in Man (OMIM), http://www.ncbi.nlm.nih.gov/Omim/ (for PITX3, MIM 602669).

\section{Authors' affiliations}

V Berry*, P K F Addison, A Moore, S S Bhattacharya, Department of Molecular Genetics, Institute of Ophthalmology, University College of London, London, UK

P K F Addison, P J Francis, A lonides, A Moore, Moorfields Eye Hospital, London, UK

Z Yang, G Karan, L Jiang, W Lin, K Zhang, Department of Ophthalmology and Visual Science, Program in Human Molecular Biology and Genetics, Eccles Institute of Human Genetics, University of Utah, Salt Lake City, UT, USA

J Hu, R Yang, Chinese Academy of Military Science, Beijing, China

*The first two authors contributed equally to this article.

This work was supported by The Wellcome Trust project grant no: $063969 / \mathrm{Z} / 01$. K Zhang is supported by grants from the National Institutes of Health (RO1EY1 4428 and RO1EY14448), American Health Assistance Foundation, the Karl Kirchgessner Foundation, The Ruth and Milton Steinbach Fund, Ronald McDonald House Charities, Macular Vision Research Foundation, Val and Edith Green Foundation, and Simmons Foundation. Z Yang is supported by grants from Fight for Sight and the Knights Templar Eye Research Foundation.

Conflict of interest: none declared.

Correspondence to: Dr V Berry, Department of Molecular Genetics, Institute of Ophthalmology, London ECIV 9EL, UK; vberry@hgmp.mrc. ac.uk

Correspondence to: Dr K Zhang, Department of Ophthalmology and Visual Science, Program in Human Molecular Biology and Genetics, Eccles Institute of Human Genetics, University of Utah, Salt Lake City, UT, USA; kang.zhang@hmbg.utah.edu

Revised version received 4 April 2004

Accepted for publication 6 April 2004

\section{REFERENCES}

1 Graw J, Loster J. Developmental genetics in ophthalmology. Ophthalmic Genet 2003;24:1-33.

2 Krumpaszky HG, Klauss V. Epidemiology of blindness and eye disease. Ophthalmologica 1996;210:1-84.

3 Evans J, Rooney C, Ashwood F, Dattani N, Wormald R. Blindness and partial sight in England and Wales: April 1990-March 1991. Health Trends 1996:28:5-12

4 lonides A, Francis P, Berry V, Mackay D, Bhattacharya S, Shiels A, Moore A. Clinical and genetic heterogeneity in autosomal dominant cataract. Br J Ophthalmol 1999:83:802-8.

5 Graw J. The genetic and molecular basis of congenital eye defects. Nat Rev Genet 2003;11:876-88

6 Semina EV, Brownell I, Mintz-Hittner HA, Murray JC, Jamrich M. Mutations in the human forkhead transcription factor FOXE3 associated with anterior segment ocular dysgenesis and cataracts. Hum Mol Genet 2001;10:231-6.

7 Azuma N, Hirakiyama A, Inoue T, Asaka A, Yamada M. Mutations of a human homologue of the Drosophila eyes absent gene (EYA1) detected in patients with congenital cataracts and ocular anterior segment anomalies. Hum Mol Genet 2000;9:363-6.

8 Hanson I, Churchill A, Love J, Axton R, Moore T, Clarke M, Meire F, van Heyningen $V$. Missense mutations in the most ancient residues of the PAX6 paired domain underlie a spectrum of human congenital eye malformations. Hum Mol Genet 1999;8:165-72.

9 Jamieson RV, Perveen R, Kerr B, Carette M, Yardley J, Heon E, Wirth MG, van Heyningen V, Donnai D, Munier F, Black GC. Domain disruption and mutation of the bZIP transcription factor, MAF, associated with cataract, ocular anterior segment dysgenesis and coloboma. Hum Mol Genet 2002;11:33-42.

10 Semina EV, Ferrell RE, Mintz-Hittner HA, Bitoun P, Alward WL, Reiter RS, Funkhauser C, Daack-Hirsch S, Murray JC. A novel homeobox gene PITX3 is mutated in families with autosomal-dominant cataracts and ASMD. Nat Genet 1998;2:167-70.

11 Lathrop GM, Lalouel JM, Julier C, OH J. Strategies for multilocus linkage analysis in humans. Proc Natl Acad Sci U S A 1984;81:3443-6. 
12 Treisman J, Harris E, Wilson D, Desplan C. The homeodomain: a new face for the helix-turn-helix? Bioessays 1992;14:145-50.

13 Richards J, Maumenee IH, Rowe S, Lovrien EW. Congenital cataract possibly linked to haptoglobin. Cytogenet Cell Genet 1984;37:570.

14 lonides AC, Berry V, Mackay DS, Moore AT, Bhattacharya SS, Shiels A. A locus for autosomal dominant posterior polar cataract on chromosome 1p. Hum Mol Genet 1997;6:47-51

15 Berry V, Francis P, Reddy MA, Collyer D, Vithana E, MacKay I, Dawson G Carey AH, Moore A, Bhattacharya SS, Quinlan RA. $\alpha$-B-crystallin gene (CRYAB) mutation causes dominant congenital posterior polar cataract in humans. Am J Hum Genet 2001;69:1141-5

16 Yamada K, Tomita H, Yoshiura K, Kondo S, Wakui K, Fukushima Y, Ikegawa S, Nakamura Y, Amemiya T, Niikawa N. An autosomal dominan posterior polar cataract locus maps to human chromosome 20p12-q12. Eur J Hum Genet 2000;7:535-9.

17 Gage PJ, Suh H, Camper SA. The bicoid-related Pitx gene family in development. Mamm Genome 1999;2:197-200.

18 Semina E, Murray JC, Reiter R, Hrstka RF, Graw J. Deletion in the promoter region and altered expression of Pitx 3 homeobox gene in aphakia mice. Hum Mol Genet 2000;9:1575-85.

19 Hittner HM, Kretzer FL, Antoszyk JH, Ferrell RE, Mehta RS. Variable expressivity of autosomal dominant anterior segment mesenchymal dysgenesis in six generations. Am J Ophthalmol 1982;93:57-70.

20 Ferrell RE, Hittner HM, Kretzer FL, Antoszyk JH. Anterior segment mesenchymal dysgenesis: probable linkage to the MNS blood group on chromosome 4. Am J Hum Genet 1982;34:245-9. 